Military Technical College Kobry El-Kobba

Cairo, Egypt

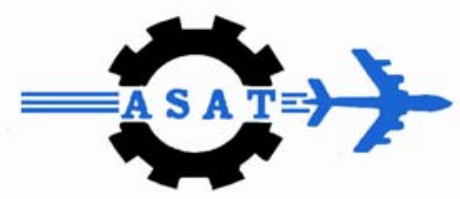

12-th International Conference

on

Aerospace Sciences \&

Aviation Technology

\title{
A MODIFIED FEC SCHEME FOR ATM QOS ENHANCEMENT OVER BURSTY SATELLITE CHANNELS
}

Amr Amin Assaad, Yahya Z. Mohasseb, and Ali Elmoghazy

\section{ABSTRACT}

In this paper the ATM Header Error Correction (HEC) mechanism was analyzed and ATM Quality of Service (QoS) parameters were calculated assuming bursty satellite channel. The use of Reed Solomon (RS) codes as an outer code to improve the QoS of ATM transmission over satellite channels was examined. The various tradeoffs which have to be made when using RS codes, such as the selection of appropriate code rates while maintaining sufficient throughput and minimal processing delay are thoroughly studied. These tradeoffs are to be used as the basis for an adaptive scheme which adapts the correction capability as well as the grouping of ATM cells into RS codewords. A Forward Error Correction (FEC) scheme is proposed which interacts with the ATM layer to alleviate the problem of excessive misinsertion of ATM cells, the performance of the proposed scheme is evaluated via simulation and is shown to significantly reduce the misinsertion rate.

\footnotetext{
* Egyptian Armed Forces
} 


\section{INTRODUCTION}

Asynchronous transfer mode (ATM) is the switching and multiplexing technique for BISDN (Broadband Integrated Services- Digital Networks) and is widely accepted as the networking technology when the quality of service is of concern. ATM technology offers users integration of voice, video, and data services. On the other hand, there is an increasing recognition of the benefits and advantages of using satellite transmission systems which play a significant role in establishing the global information infrastructure. With the increasing demand for ATM services, satellite networks will play an important role in the development of ATM networks [1].

Satellite ATM networks have significant advantages over terrestrial ATM networks such as remote coverage with rapid deployment, distance insensitivity, bandwidth on demand, immunity to terrestrial disasters, and supporting broadband links [1-2].

Since ATM was originally designed for optical fiber media with excellent error characteristics, several adaptations are needed in order to apply ATM to the adverse satellite channel with higher error probability. The noise process affecting the satellite link is considered to be a pure Additive White Gaussian Noise (AWGN) process. A satellite link is mostly stationary and hence there is virtually no fading (except for land mobile satellites). Alternatively, the satellite channel may be modeled as exhibiting error bursts which is modeled by an additional noise process [3]. This process is considered a finite duration Gaussian noise pulse whose occurrence follows a Poisson distribution [3].

Many solutions were proposed to overcome the bursty problem. These solutions include modifying the ATM cell structure by increasing the correction capability of the HEC [4], introducing Automatic Repeat Request (ARQ) mechanisms [5], using interleaving mechanisms [6] and FEC [7]. Modification of the ATM HEC requires a dedicated gateway to achieve compatibility with terrestrial links. ARQ schemes increase delay and are not suitable for real time traffic. Interleaving causes a decoding delay which depends on interleaving depth. Therefore this paper concentrates on the use of FEC to reduce the BER. This solution has the added advantage of reducing the transmission power needed to achieve a certain QoS. Several FEC schemes have been proposed to remedy this problem [8]. Many schemes have focused on Reed Solomon (RS) codes due to their non binary structure and maximum distance separable properties [9]. These properties make RS codes better suited for bursty error correction. Using these codes adaptively was studied in literature for two predominant reasons. First, adaptation according to the channel conditions [6] which may be relayed back the transmitter through any of feedback channel. The second is adaptation according to Quality of Service QoS required by different types of data [10], thus maximizing the channel utilization, throughput and application flexibility.

In this paper, the effect of applying FEC to ATM cells on the ATM QoS parameters was studied. First, we quantify the effect of a simple concatenation of the ATM cells with RS FEC. Based on the simulation results of this simple concatenation scheme, we propose an improved scheme. This scheme utilizes extra information available from the RS decoder in order to improve the misinsertion rate which is the most 
critical QoS parameter. Results for the proposed scheme show a decrease in misinsertion rate at the expense of a slightly higher Cell Loss Rate (CLR).

Finally, a discussion of the various tradeoffs associated with adaptive schemes is presented. The presented simulation results are useful in predicting the performance and selecting the appropriate code rate and error correction capability for any adaptive scheme based on the studied RS codes.

Following this introduction, Section II outlines the simulation model. Section III quantifies the effect of concatenating of the ATM cells with RS FEC. The proposed scheme is presented in Section IV. Section V discusses of the various tradeoffs associated with adaptive schemes. Finally Section VI offers concluding remarks.

\section{SIMULATION MODEL}

In order to evaluate the performance of coded ATM cells with various coding and channel parameters MATLAB software was developed to simulate system depicted in Figure 1. The information source generates equiprobable random binary digits. These random bits are segmented into blocks of 384 bits (48 bytes) representing the payload of the ATM cells. To simulate header information, 4 bytes are randomly generated and used to calculate the HEC bytes.

This cell generation process simulates the function of the AAL layer in segmenting the payload and the function of the ATM layer in forming the ATM cell. ATM cells then passed to the RS encoder. Several cells may be grouped into one RS codeword to minimize decoding delays. Further discussion of the effects of cell grouping will be presented in later sections. The RS encoded ATM cells are then modulated prior to transmission. Since the aim of the simulation is to study the interaction between the FEC process and the ATM QoS, rather than quantifying the exact performance parameters, for certain $E_{b} / N_{t}$, BPSK modulation was chosen for simplicity. A more practical multilevel modulation scheme will not change the relative behaviors of the used schemes.

The data is then transmitted through the channel which can be either AWGN or bursty satellite channel. To simulate the bursty satellite channel, the model presented in [3] is used. This model represents the bursty nature of the channel the sum of two noise processes, namely, the back ground Gaussian noise $n_{g}(t)$ and the burst noise $n_{b}(t)$. The summation of the background Gaussian noise and burst noise is referred to as bursty noise $n_{d}(t)$.

$n_{d}(t)=n_{g}(t)+n_{b}(t)$

In order to describe the burst-noise component of the channel noise, let $a(t)$ denote a sample function from a delta-correlated Gaussian stochastic process with zero mean and double-sided power spectral density (PSD) $N_{b} / 2$ and let $t_{i}$ denote a set of Poisson points with average arrival rate $v$. The burst noise component becomes:

$n_{b}(t)=a(t) \sum_{i=-\infty}^{\infty} \Pi\left(\frac{t-t_{i}-d / 2}{d}\right)$ 
Where $\Pi(t / d)$ is a unit-amplitude pulse of width $d$ centered at $t=0$. The normalized average power for the burst noise process is given by $\left(v d N_{b} / 2\right)$

The background Gaussian-noise component $n_{g}(t)$ is assumed to be zero-mean and delta correlated with normalized average power given by $\left(N_{g} / 2\right)$. Note that the two noise processes are uncorrelated, the total noise power is given by the sum of the variances of the two components that is:

$\operatorname{var}\left(n_{g}(t)\right)+\operatorname{var}\left(n_{b}(t)\right)=\left(N_{g}+N_{b} v d\right) / 2=N_{t} / 2$

This model is parameterized by the ratio of the burst noise power to the total noise power which is given by:

$\psi=\frac{v d N_{b}}{N_{g}+v d N_{b}}$

The inverse of the operations executed in the transmitter are performed in the receiver, beginning with demodulation then the decoding by the $\mathrm{RS}$ decoder using Berlekamp algorithm [9]. The data is then passed to the ATM layer where the header is separated and the HEC is calculated. Accordingly the cells are either discarded or delivered to the higher layers. In the simulation scenario, discarded cells will be counted to determine the cell loss rate. Moreover, other QoS parameters such as misinsertion rate or cell error rate are computed by checking the received ATM cell against the transmitted cells.

As will be shown in Section $V$, performance can be enhanced by utilizing the intrinsic information available at the RS decoder. This proposal is shown dotted in Figure 1.

\section{II.A Performance Analysis}

All ATM QoS parameters are based on the outcomes of the HEC which is standardized in the ITU-T [11] and ATM Forum. HEC can correct single bit errors. It detects certain multiple bit errors that is only employed in the ATM cell header and not in the cell payload. HEC operates in two modes: the correction mode and the detection mode. In the correction mode, a single-bit error can be corrected and cells with multiple-bit errors are discarded. In the detection mode, all cells with detected errors in the header are discarded.

Performance is expressed through three QoS paramters; two types refer to cells with error in the header which are Cell Loss Rate (CLR) and Cell Misinsertion Rate (CMR) and one type refer to cells with error in the payload which is Cell Error Rate (CER).

A cell is discarded if the receiver is in correction mode and more than one error occurs or if the receiver is in detection mode and at least one error occurs.

Hence, the probability of cell loss rate is given by

$C L R=P_{c} . P(>1$ error in the header $)+P_{d} . P(\geq 1$ error in the header $)$

$P_{c}$ and $P_{d}$ are the probabilities that the receiver is in correction mode and in detection mode, respectively. These two probabilities can be computed by modeling the dual mode operation of the HEC mechanism as a Markov chain with two states [9]. Solving the state equations yields

$C L R=P(0) \cdot[1-P(0)-P(1)]+[1-P(0)] .[1-P(0)]$

Where $P(0)=(1-P)^{40}$ and $P(1)=40 . P .(1-P)^{39}$ and $P$ is the channel bit error rate The probability of cell error rate is given by 
$C E R=P(\geq 1$ error in the information field $)=1-(1-P)^{i}$

Where $i=384$ is the number of information bits in the ATM payload.

For the uncoded case $P$ for AWGN is given by

$P=Q\left(\sqrt{2 \frac{E_{b}}{N_{t}}}\right)$

and for bursty satellite channel is given by [3]:

$P=Q\left(\sqrt{\frac{2}{(1-\psi)}\left(\frac{E_{b}}{N_{t}}\right)}\right)(1-v d)+Q\left(\sqrt{\frac{2}{1-\psi+\frac{\psi}{v d}}\left(\frac{E_{b}}{N_{t}}\right)}\right)(v d)$

Where $Q(x)=\frac{1}{\sqrt{2 \pi}} \int_{x}^{\infty} e^{-\beta^{2} / 2} d \beta$, These equations are used for validation of simulation model. However in the reminder of the paper we focus on coded performance.

\section{CODED PERFORMANCE OF ATM CELLS}

In this section the performance of the HEC concatenated with various RS codes will be discussed in terms of CER, CLR and CMR in AWGN and bursty channels. No interaction between the physical layer (RS encoding) and the ATM layer (HEC) is assumed at this point. To avoid breaking the ATM cell into multiple code blocks, all RS codes are shortened to ensure that the message bits are multiple of 53 bytes.

Shortened codes have the same hardware complexity and correction capability as the codes from which they are derived but with increased code rate [12]. A shortened RS $\left(n_{1}, k_{1}\right)$ implicitly uses an $(n, k)$ encoder, where $n=2^{m}-1$ and $k=k_{1}+\left(n-n_{1}\right)$, and the generator polynomial for the mother and shortened codes are the same [9].

Emphasis is made on the shortened RS codes: RS(57,53), RS(59,53), RS(61,53), $\mathrm{RS}(63,53)$, and $\mathrm{RS}(65,53)$ under $\mathrm{GF}\left(2^{8}\right)$. Their capability $t$ correspond to $2,3,4,5$ and 6 symbol, respectively. Preserving cell structure precludes the need for delay in RS encoder to wait for further cells to add to the RS codeword. Delay can also be eliminated by padding with zero cells. However this wastes bandwidth. Other choices of RS codes with multiple cells per RS codeword will be discussed in Section VI.

Figure 2 shows the results of CLR vs. $E_{b} / N_{t}$ using standard HEC concatenated with the RS and also shows the simulated and theoretical (equations5,6) results for uncoded HEC in AWGN for comparison. Figure 3 shows the CLR vs. $E_{b} / N_{t}$ when the HEC is concatenated with the RS code with different capabilities in bursty channel. It is noticed that as the capability of the RS code increases, CLR decreases. However, the coding gain for CLR with the RS coding in the bursty channel is more than in the AWGN. For example, the RS $(65,53)$ provide a coding gain of $2 \mathrm{~dB}$ at $C L R=10^{-4}$ for the AWGN channel. The same code has a coding gain of $4 \mathrm{~dB}$ for the same $C L R$ under bursty satellite channel. This confirms that the RS is more suitable in bursty than AWGN. This suitability results from the fact that the correction capability is measured in symbols rather than bits. Thus, multiple adjacent bit errors 
occurring within the same symbol count as one error only. Therefore the RS code performs better when correcting adjacent errors.

Figure 4 shows the CER vs. $E_{b} / N_{t}$ in AWGN and also the theoretical result for uncoded CER for comparison. The CER is concerned with the information and not the header, so the HEC has no role in its calculations. As expected, it is noticed that as the capability $t$ of the RS code increases, CER decrease. Figure 5 shows similar results but in bursty channel. As in CLR performance it is noticed that as the capability of the RS code ${ }^{t}$ increases, CER decrease, and also the coding gain of CER with the RS in the bursty channel is more than in the AWGN. For example, the $\mathrm{RS}(65,53)$ provide a coding gain of $4 \mathrm{~dB}$ at $C E R=10^{-4}$ for the AWGN channel. The same code has a coding gain of $6 \mathrm{~dB}$ for the same CER under bursty satellite channel.

Figures 6 and 7 show by simulation the relation between $E_{b} / N_{t}$ and the misinsertion rate when the HEC is concatenated with the shortened RS codes over GF $\left(2^{8}\right)$ stated before in AWGN and bursty channel respectively. It is noticed that the coding gain of misinsertion rate in the bursty channel with RS codes is more than AWGN. For example, the RS $(65,53)$ provide a coding gain of $2.5 \mathrm{~dB}$ at $C M R=10^{-3}$ for the AWGN channel. The same code has a coding gain of $4 \mathrm{~dB}$ for the same $C M R$ under bursty satellite channel. It is worth noting that the interaction between the two modes of the $\mathrm{HEC}$, detection and correction, results in the increase of CMR with $E_{b} / N_{t}$ to a certain value, and then it begins to decrease as shown in Figure 6. A detailed explanation of this phenomena was provided in [13].

\section{PROPOSED SCHEME (MARKING HIGHLY ERRONEOUS CELLS)}

The misinsertion rate is the most critical QoS. It is named catastrophic error because the receiver consideres the information to be a valid cell while actually it is ministered cell. In the previous scheme, the ATM layer receives the ATM cell after the RS decoder, even when the number of errors is beyond the correction capability of the RS decoder. According to the Berlekamp algorithm, when the order of the error polynomial is found to be higher than the correction capability, the decoder outputs the codeword as-is (after stripping the parity bits). So extra information is available from the RS decoder, this information can provide improvements if it is passed to the ATM layer to prevent misinsertion.

Hence, we propose that the RS decoder passes this information to the ATM layer with the un-correctted cell. Instead of checking the HEC as usual the ATM layer will simply discard the cell without checking. The effect of this action is two fold. First, if most of errors are in the payload, and the HEC at most contains at most one error, this will prevent cell error. Second, if the errors are concentrated in the header but are beyond the detection of the HEC then this will prevent misinsertion but will increase CLR. The reasoning behind this approach is that an increase in CLR is far better than an increase in misinsertion rate which consumes the resources and bandwidth in higher layers. The proposed scheme was applied to the AWGN and the bursty channels. 
Figure 8 shows the misinsertion rate results for the AWGN channel with the shortened RS codes RS $(61,53)$ and RS $(63,53)$. It can be seen that the proposed scheme provides a gain of about $1.3 \mathrm{~dB}$ at $10^{-3}$ probability of error for $t=4$ correction capability and $1.8 \mathrm{~dB}$ at $10^{-4}$ for $t=5$ correction capability. Figure 9 shows the CLR performance for the same two codes. It is clear that the price paid for misinsertion rate improvement is CLR increase with respect to the previous scheme. It is an acceptable price since the misinsertion rate is the catastrophic error.

It should be noted from the Figures 8,9 that the improvement in misinsertion is more evident at lower $E_{b} / N_{t}$. This intuitive result is due to the reduced bit error rate at higher $E_{b} / N_{t}$. This reduced bit error rate retains the errors of the decoded RS codewords within the capabilities of the HEC. Hence, the impact of the proposed scheme is negligible in both its positive aspects (misinsertion rate improvement) and its price (CLR increase).

Figures 10 and 11 show the results of the proposed scheme in bursty channel for the misinsertion rate and the cell loss rate respectively. It is clear that the proposed scheme provides a gain of about $1.5 \mathrm{~dB}$ at $10^{-3}$ probability of error and $1 \mathrm{~dB}$ at $10^{-4}$ for $t=3$ correction capability and $1.6 \mathrm{~dB}$ at $10^{-4}$ for $t=4$. It is important to note that the misinsertion rate of a $t=3$ scheme under the proposed scheme is superior to that of $t=5$ under the conventional simple concatenation approach. This enables the use of a higher code rate for a given target QoS and hence provides bandwidth savings. In bursty channel we choose the shortened RS codes RS $(59,53)$ and RS $(61,53)$. Also the misinsertion rate decrease and the CLR increase with respect to the previous conventional scheme as shown in Figures 10 and 11.

\section{ADAPTIVE RS CODES}

Up to this point it was assumed the RS encoder process one cell at a time. That is, each codeword corresponds to a single ATM cell. However, it should be noted that all the codes discussed so far are shortened from the mother code over $\operatorname{GF}\left(2^{8}\right)$. This imposes a complexity limit on the encoding and decoding operations. This complexity changes only marginally if the error correction capability is changed or if no shortening is applied. Furthermore, if the system is allowed to use different versions of the same mother code (i.e. different shortenings \& different correction capabilities) Then this could be used to match the encoding scheme to either the type of data or the channel conditions. The former was discussed in [10] and the latter in [6].

In this section the tradeoffs associated with the complexity, code rate, bandwidth utilization and correction capabilities are quantified. Note that the specifics of the adaptation algorithm are not discussed. This includes the feedback algorithm, identification of payload type by RS encoders, and notifying the receiver of code rate changes. Emphasis is on the impact of varying code parameters on performance.

A shortened RS code is denoted by three parameters e.g. $(255, k, \ell)$. The additional parameter $\ell$ represents the number of shortening symbols. For example, $(255,245,192)$ represents a shortening of the mother code $(255,245)$ by 192 symbols 
to become $(63,53)$. To guarantee that all codewords will contain an integer number of ATM cells, we focus on shortened codes that have $i * 53$ information symbols. Different correction capabilities are studied. This result in the codes being used having the form $(i * 53+2 t, i * 53)$. An adaptation algorithm may change $i$ between 1 and 4 , we focus on the two boundary ( $1 \& 4)$ since the performance of $i=2$ or $i=3$ will be in between those two cases. Values of $t$ in the following discussion are 4, 5 and 16. Values of $i$ in excess of 4 require a change in the mother code.

Figures 12 and 13 show the CER performance of AWGN and bursty characteristics respectively for different code rates and correction capabilities. Starting from the RS $(61,53)$ code which uses one ATM cell per code word and has a correction capability of 4 bytes we see that if we group 4 ATM cells into one codeword while matching the same correction capability using $\mathrm{RS}(220,212)$, there is a loss of $1.3 \mathrm{~dB}$ at $10^{-3}$ for AWGN channel and of $1.1 \mathrm{~dB}$ for bursty channel. However, in this case the $\mathrm{RS}(220,212)$ code has the advantage of increasing the code rate from 0.8688 to 0.963 which increase the throughput with the same amount. This may be beneficial in the case of real time application where performance is largely dependent on the throughput rather than occasional errors in cells. In contrast if the system designer chooses to vary the channel bit rate to maintain a constant throughput then, the choice of $\mathrm{RS}(220,212)$ reduces bandwidth requirements. Furthermore, since both codes are derivatives from GF $\left(2^{8}\right)$, the decoding delay per codeword is the same for both codes. Since the RS $(220,212)$ has 4 ATM cells per codeword this reduces the latency in the channel. The loss in the CER performance is a direct result of using the same correction capability to correct a larger number of symbols. Results for $t=5$ corresponding to the code $\mathrm{RS}(63,53)$ and $\mathrm{RS}(222,212)$ are also shown in the figures, the loss in the CER is $1.2 \mathrm{~dB}$ for AWGN at $10^{-2}$ and $1.4 \mathrm{~dB}$ for bursty at $10^{-2}$.

To compare results with the same average correction capability per ATM cell, the RS $(244,212)$ with of $t=16$ symbols is examined. On average, this code will also correct 4 symbol errors per ATM cells. Furthermore it has exactly the same code rate and bandwidth utilization as the RS(61,53) code. Figures 12 and 13 show that the CER performance of this $\operatorname{RS}(244,212)$ code is superior to both the $\operatorname{RS}(220,212)$ and RS $(61,53)$ codes. In particular there is a gain of $2.1 \mathrm{~dB}$ over $\operatorname{RS}(220,212)$ and $0.8 \mathrm{~dB}$ over RS(61,53) at $10^{-3}$ probability of error in AWGN and there is a gain of $2 \mathrm{~dB}$ over $\mathrm{RS}(61,53)$ and $3 \mathrm{~dB}$ over $\mathrm{RS}(220,212)$ at $10^{-3}$ probability of error bursty respectively.

Although both the RS $(61,53)$ and RS $(244,212)$ correct the same average number of symbols per cell, RS $(244,212)$ provides $0.8 \mathrm{~dB}$ performance advantage in AWGN at $10^{-3}$. To explain this, assume a burst of 12 symbol errors occur within the first 2 cells and no errors occur for the following 2 cells. For the RS( 61,53) this results in 2 cells errors since the number of errors are beyond its correction capability. However, the $\mathrm{RS}(244,212)$ is can correct 12 errors and the result is four valid cells.

\section{CONCLUSION}

The effect of applying Forward Error Correction (FEC) to ATM cells on the quality of service parameters was studied, and these results were compared to the effect of the HEC only on the ATM without any FEC. First, we quantify the effect of a simple 
concatenation of the ATM cells with RS FEC. Based on the simulation results of this simple concatenation scheme, we propose an improved scheme. This scheme utilizes extra information available from the RS decoder in order to improve the misinsertion rate which is the most critical quality of service parameter. Results for the proposed scheme show a decrease in misinsertion rate at the expense of a slightly higher Cell Loss Rate (CLR). Finally, a discussion of the various tradeoffs associated with adaptive schemes was presented. These results are useful in predicting the performance and selecting the appropriate code rate and error correction capability for any adaptive scheme based on the studied RS codes.

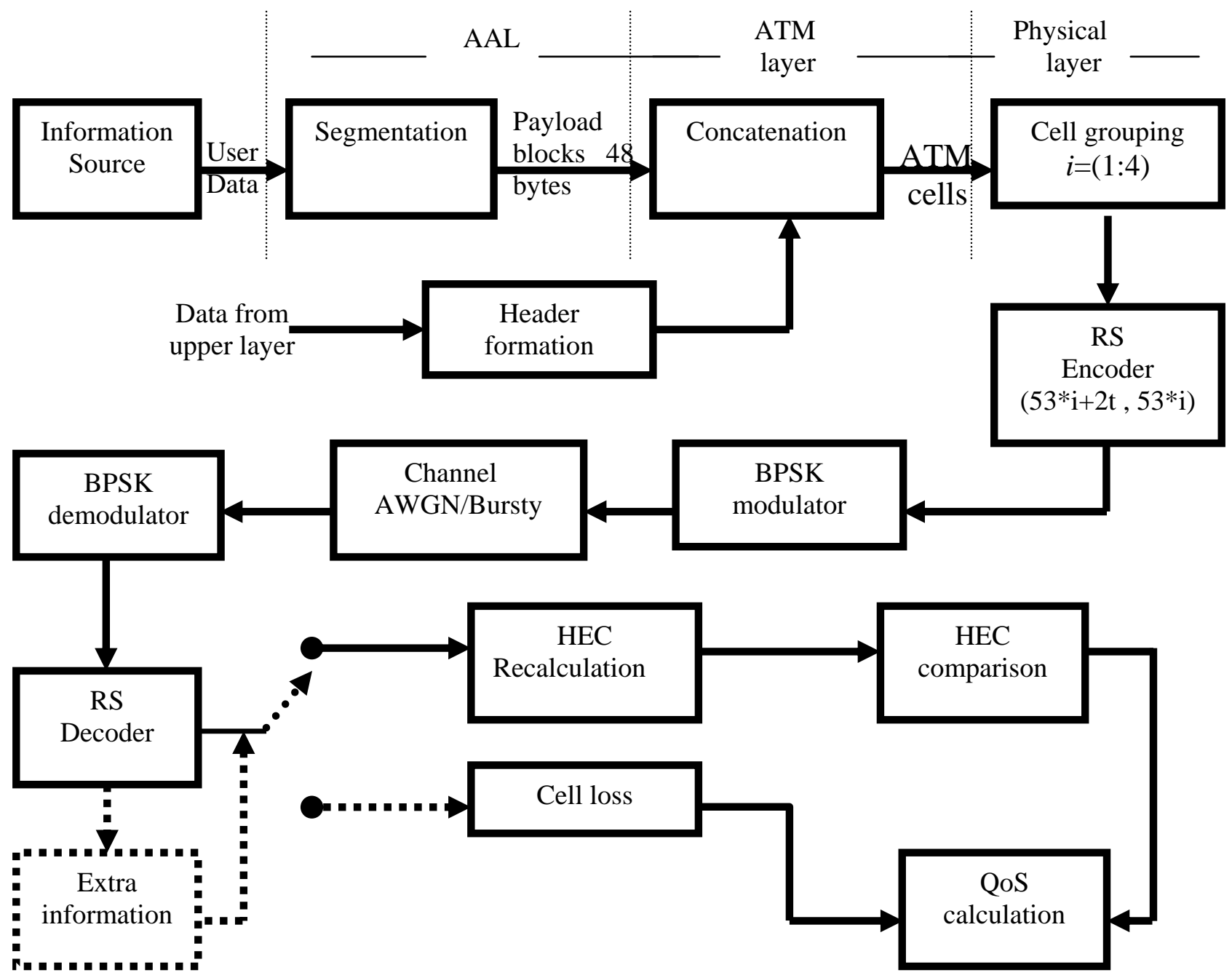

Figure 1. Simulation Model 


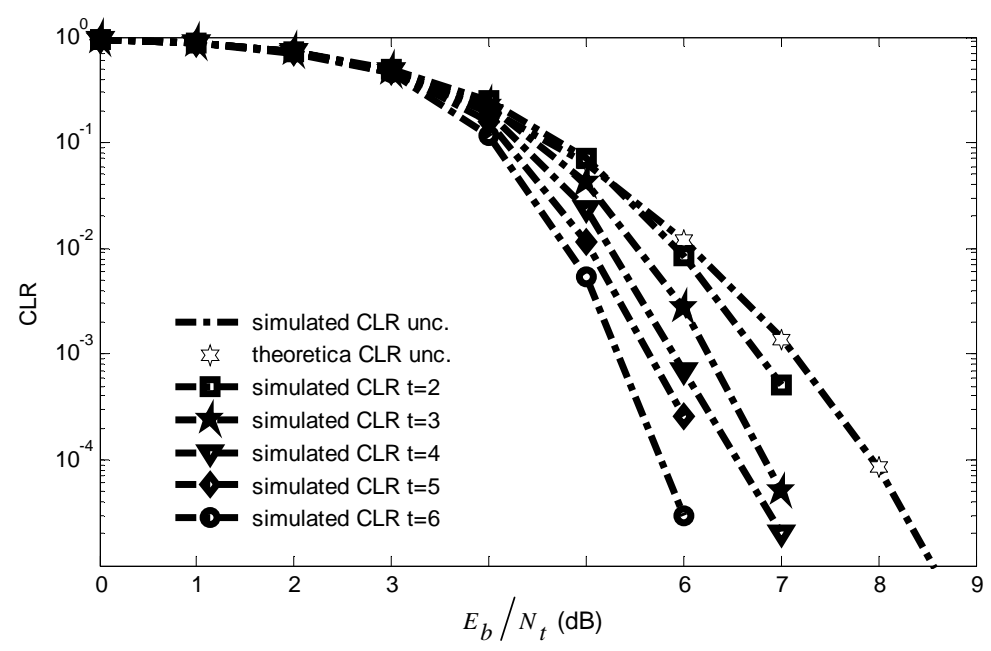

Figure 2 Performance of coded ATM cell through CLR in AWGN

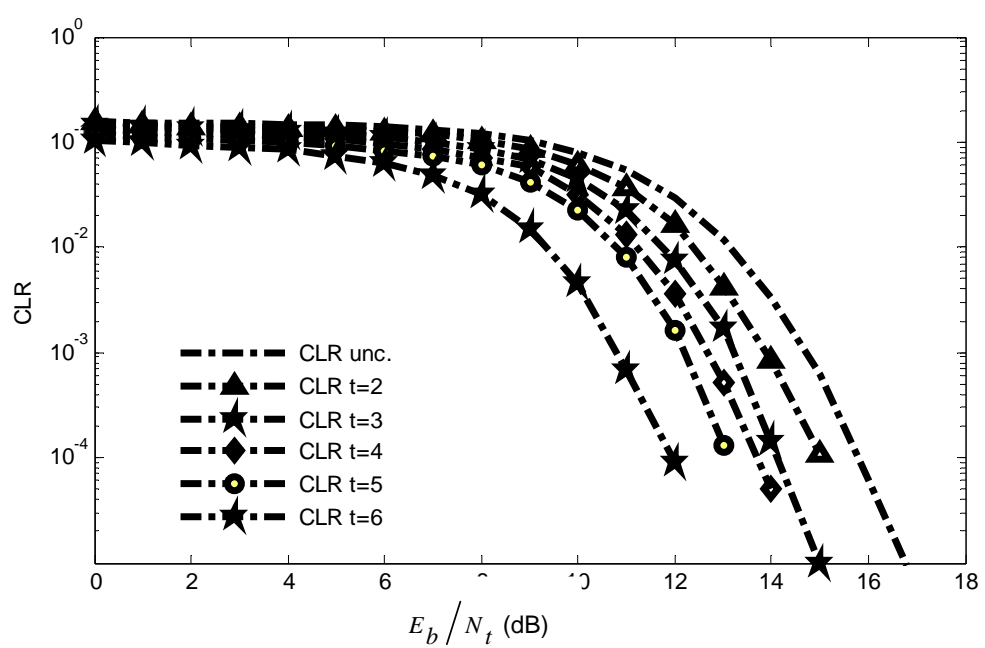

Figure 3 performance of coded ATM through CLR in bursty channel $(\psi=0.8 v d=0.1)$

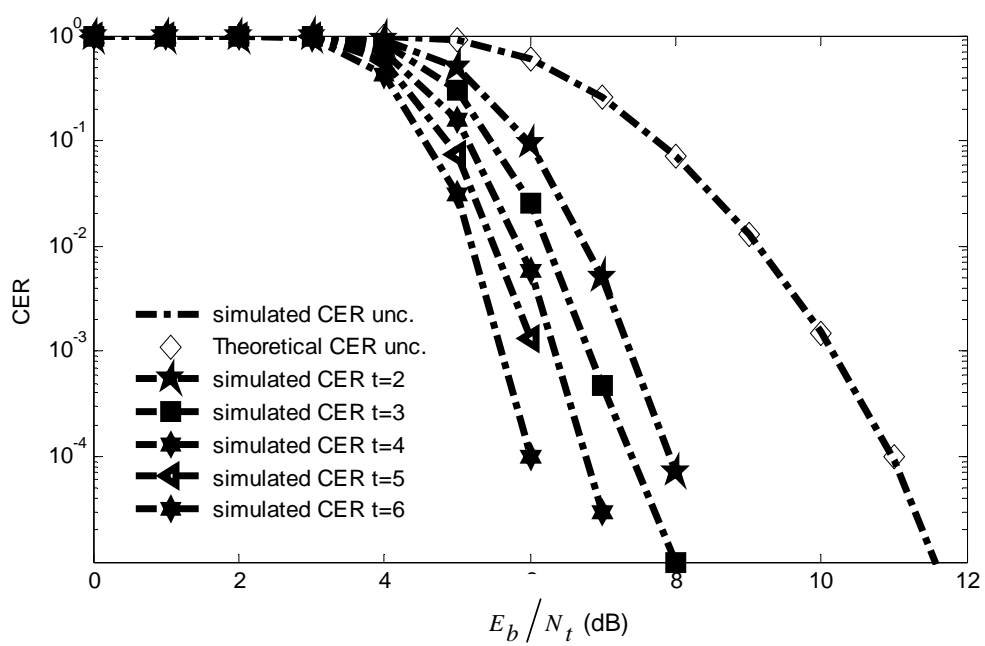

Figure 4 Performance of coded ATM cell through CER in AWGN 


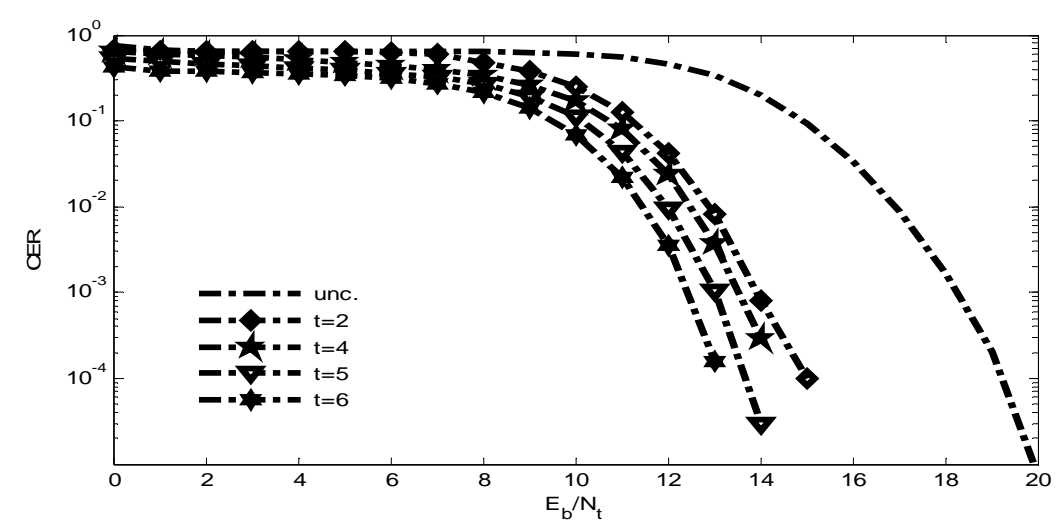

Figure 5 Performance of coded ATM cell through CER in bursty channel $\left(\psi=E_{b} / N_{t}(\mathrm{~dB}) 1\right)$

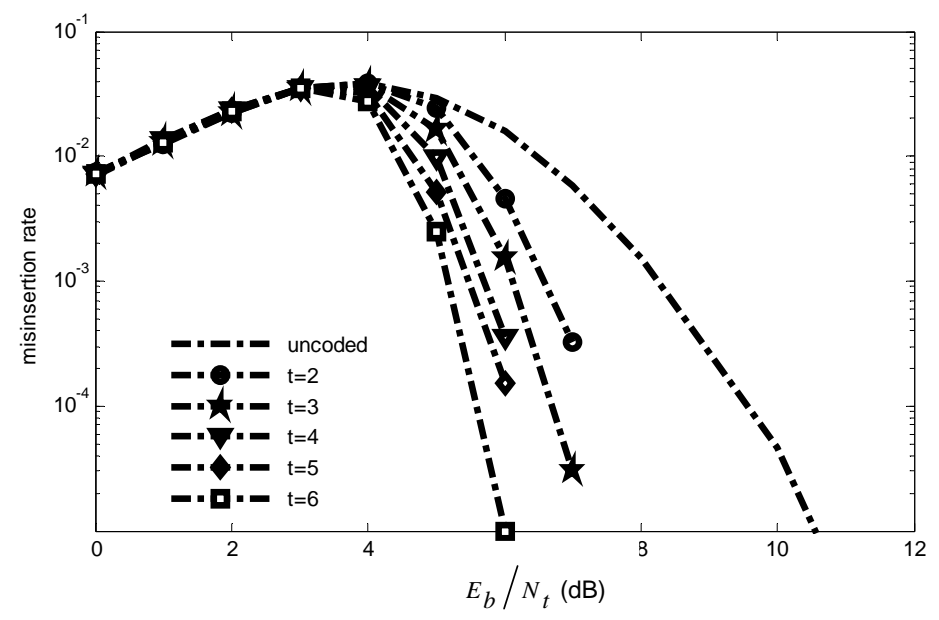

Figure 6 Performance of coded ATM cell through in AWGN channel

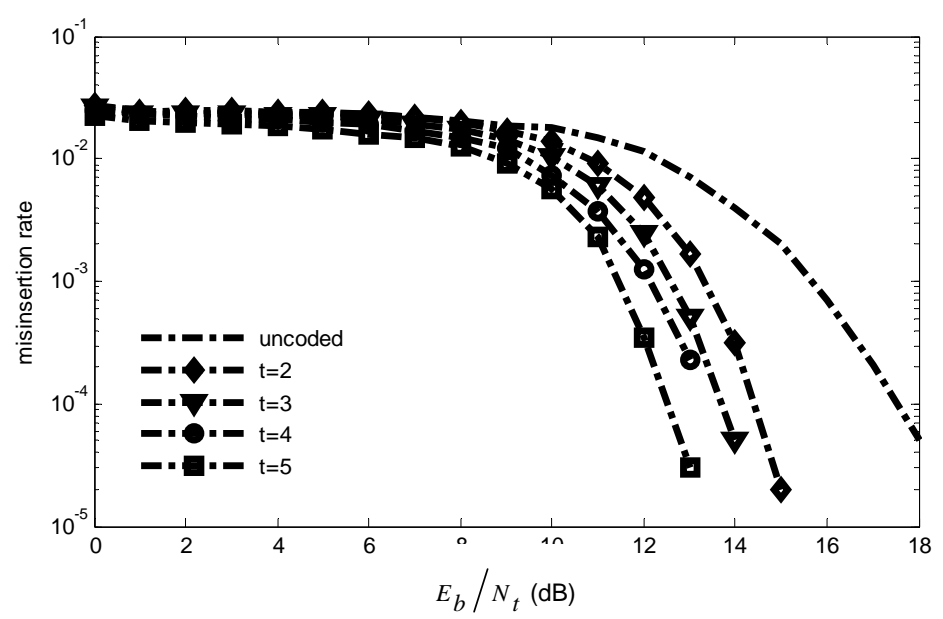

Figure 7 Performance of coded ATM cell through misinsertion rate in bursty channel $(\psi=0.8 v d=0.1)$ 


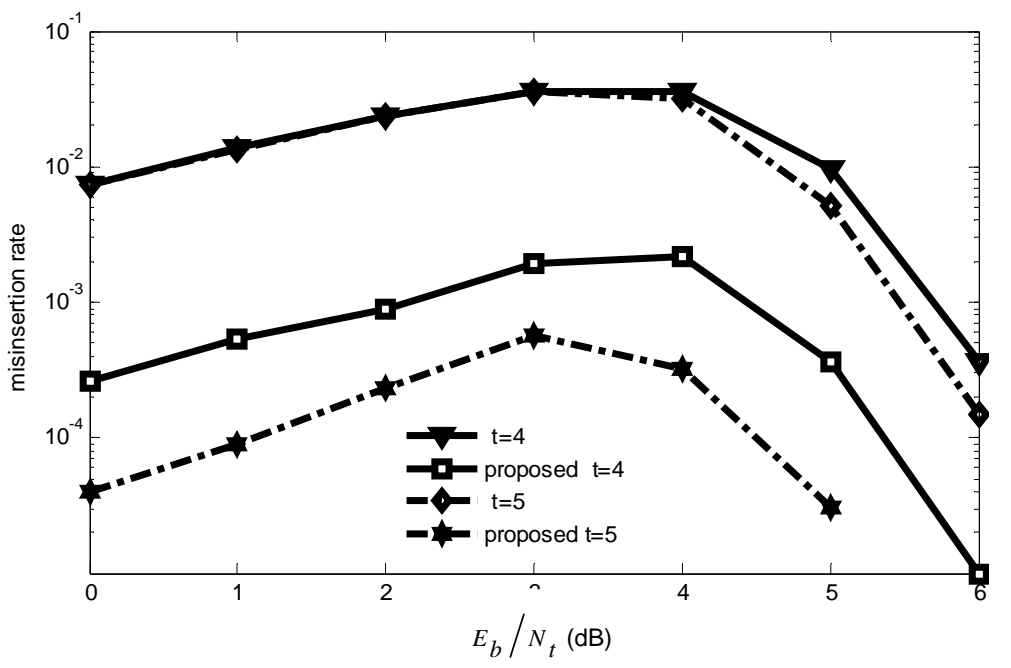

Figure 8 Performance of coded ATM cell through misinsertion rate in AWGN channel with the proposed scheme

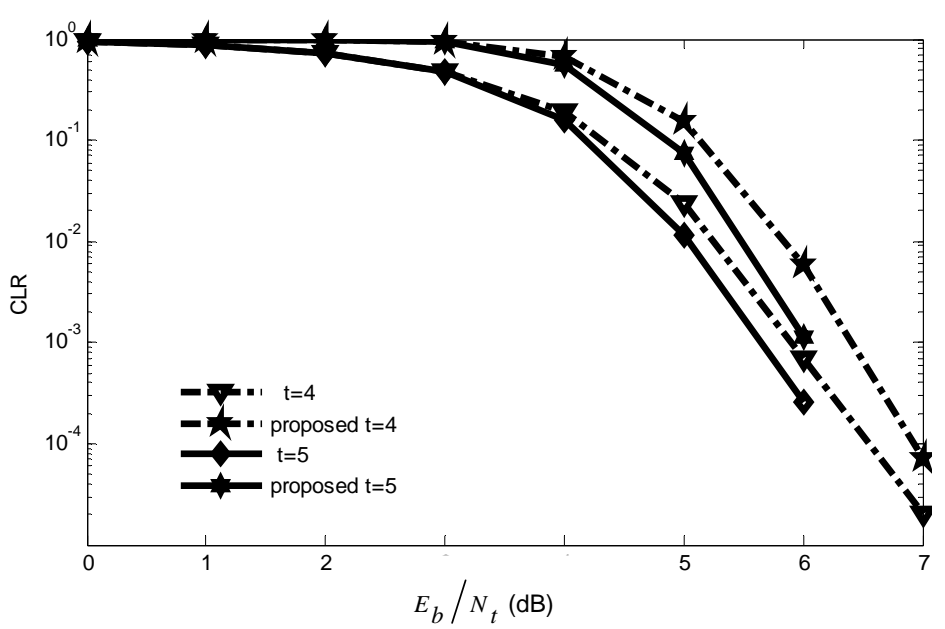

Figure 9 Performance of coded ATM cell through CLR in AWGN channel with the proposed scheme

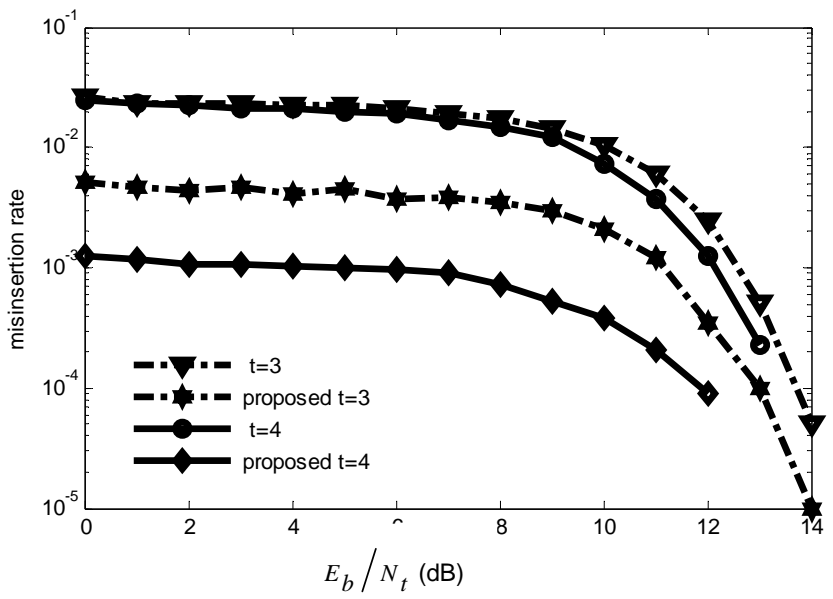


Figure 10 Performance of coded ATM cell through misinsertion rate in bursty channel with the proposed scheme $(\psi=0.8, v d=0.1)$

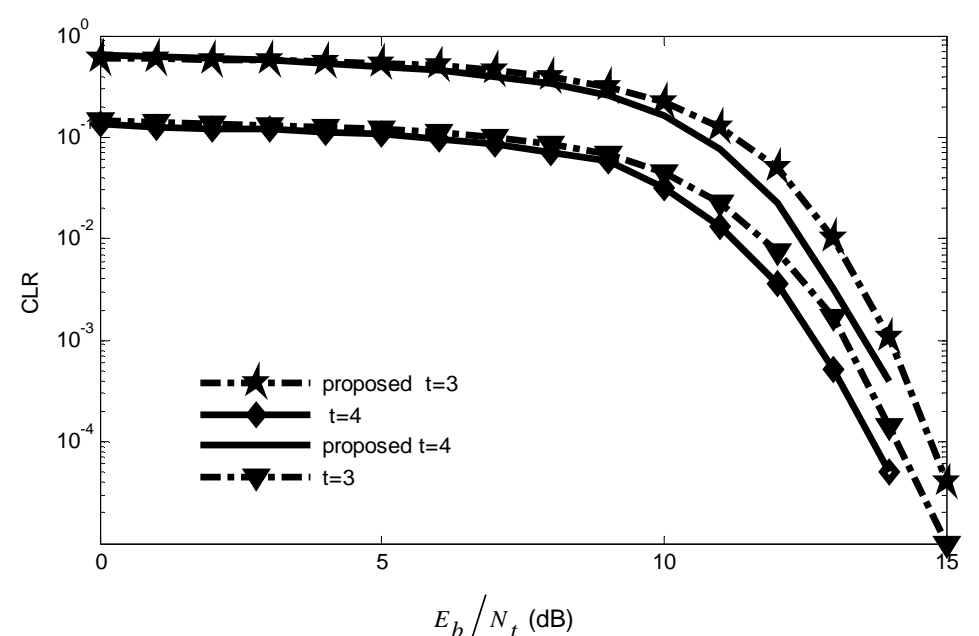

Figure 11 Performance of coded ATM cell through CLR in bursty channel with the proposed scheme $(\psi=0.8, v d=0.1)$

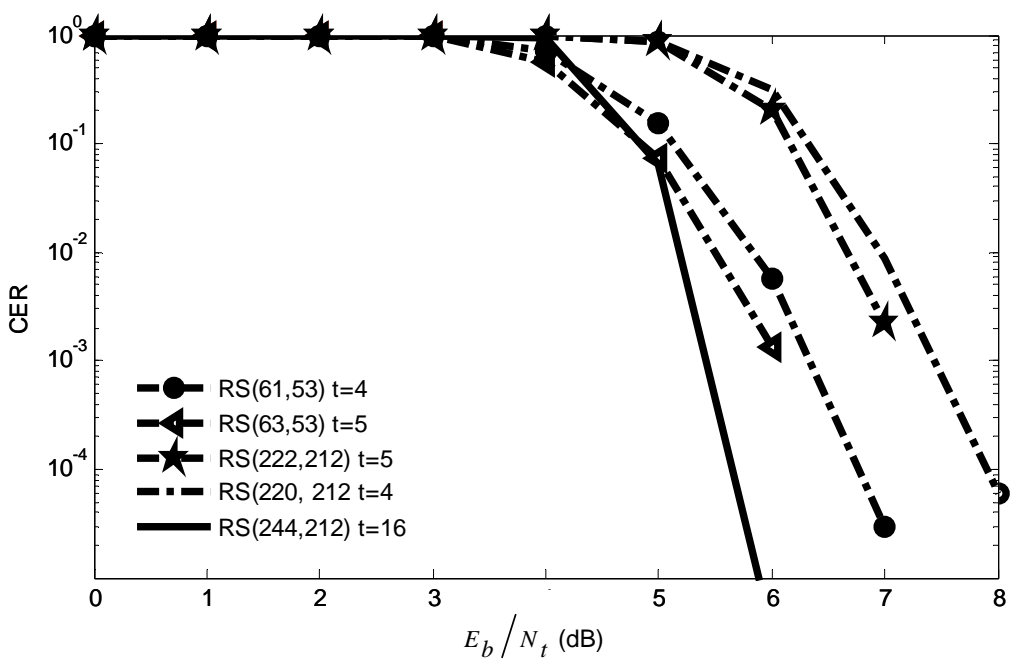

Figure 12 Performance of coded ATM cell through CER in AWGN channel with the adaptive scheme. 


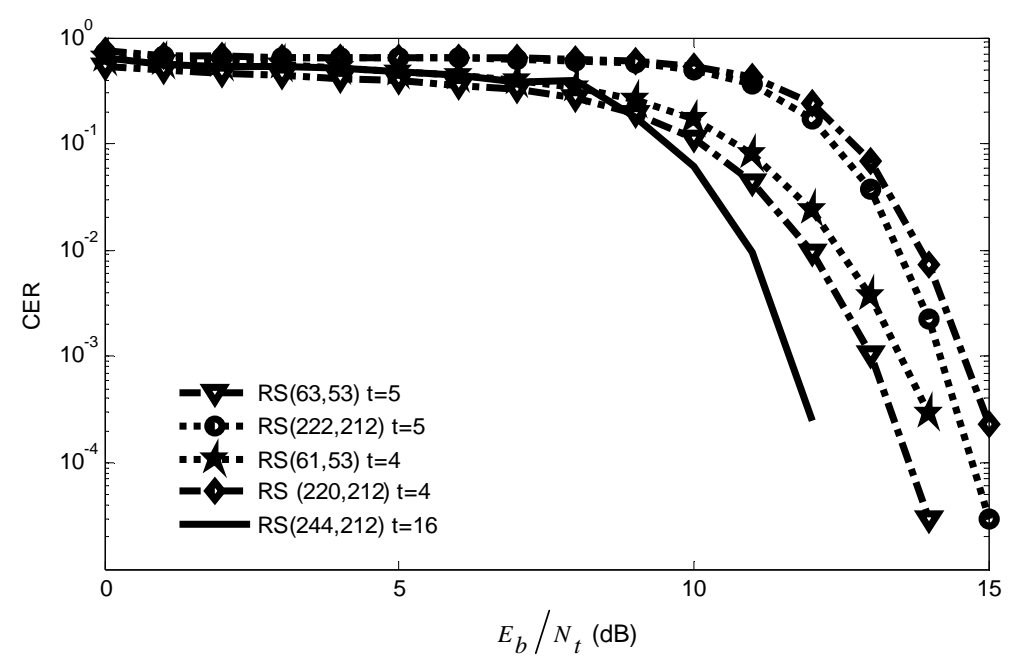

Figure 13 Performance of coded ATM cell through CER in bursty channel with the adaptive scheme

\section{References}

[1] I. F. Akyildiz, S. Jeong, "Satellite ATM Networks: A Survey", IEEE Communications Magazine, Vol. 35, No. 7, pp. 30-43, July 1997.

[2] Sastri Kota and Raj Jain " Satellite ATM Network Architurectural Considerations and TCPIIP Performance " proceeding of the 3rd Ka Band Utilization Conference, Sorrento, pp. 55-58, Italy September 1997.

[3] William J. Ebel, William H. Tranter, "The Performance of Reed-Solomon Codes on a Bursty-Noise Channel," IEEE Transactions on Communications, Vol. 43, No. 2/3/4. pp. 298-306, February /March /April 1995.

[4] S. Aikawa, "Forward error correction schemes for wireless ATM systems", Proc. IEEE ICC '96 (1996).

[5] A. Hmimy, C.Gupta, "Throughput Analysis Of ATM over Satellite using ARQ and Adaptive Concatenated FEC Codes," IEEE Emerging Technologies Symposium: Broadband, Wireless Internet Access, 2000. 
[6] K. Wang, Bin Tian, K. Yi, "A Practical Scheme of Improving the Link Performance for Satellite Communication Networks," Proceedings of the 17th International Conference on Advanced Information Networking and Applications, 2003.

[7] Y. A. Tesfai, S.G Wilson, "FEC schemes for ATM traffic over wireless links," pp. 948-953, Proc. Milcom 96.

[8] J. Bibb Cain, D. McGregor, "A Recommended Error Control Architecture for ATM Networks With Wireless Links," IEEE Journal on Selected Areas in Communications, Vol. 15, No. 1, pp.16-28, January 1997.

[9] S. B. Wicker, "Error Control Systems for Digital Communication and Storage," Prentice-Hall, 1995.

[10] Li, S.J. , Pan, K.F. , Yuan, J.S. , Vigil, A.J. , Berg, A. "Adaptive Reed-Solomon coding for wireless ATM communication" Proceedings of the IEEE Southeastcon pp:27 - $30,2000$.

[11] "B-ISDN user-network interface- Physical layer specification General characteristic," ITU-T I.421 (1999).

[12] S. Lin and D.J. Costello Jr, "Error Control Coding Fundamentals and Applications," Prentice-Hall, 2004.

[13] Amr Assaad, "Satellite ATM Communication Links," Master Thesis, The Military Technical College, Cairo 2007. 